\title{
The effect of osteogenic differentiation of Periodontal Ligament Stem Cells isolated from the periodontium of healthy teeth and periodontitis-affected teeth induced by SDF-1
}

\author{
Fengzhi Wang ${ }^{1, a}$, Jianmin $\mathrm{He}^{2,{ }^{*} b}$ \\ ${ }^{1}$ School of Stomatology Lanzhou University, Lanzhou, Gansu 730000, China; \\ 2 Department of Stomatology, Gansu Provincial Hospital, Lanzhou 730000, China. \\ ${ }^{a} 754404385,{ }^{*}$ Corresponding author Email: ${ }^{b}$ wangfengzhi556@163.com
}

Keywords: Periodontitis;Regeneration, Stem Cells, Stromal cell derived factor-1, health Periodontal ligament stem cells, inflammatory Periodontal ligament stem cells, osteogenic differentiation.

\begin{abstract}
Stem cell therapy is currently a hot topic in tissue regeneration. This study aimed to compare the the effect of osteogenic differentiation and proliferation ability of health Periodontal ligament stem cells (hPDLSCs) and inflammatory periodontal ligament stem cells (iPDLSCs) with SDF-1 that isolated from the periodontium of healthy and periodontitis-affected teeth.
\end{abstract}

\section{Introduction}

Periodontitis is an inflammatory disease which manifests clinically as loss of supporting tissues and leads to tooth loss[1].In periodontitis, the main goal of treatment is to prevent supporting periodontal tissues from lossing and make the damaged tissue regeneration.Possible applications of Stem cells (SCs) for repair and regeneration of damaged tissues are now the focus of attention of many researchers as they have the ability for indefinite self-renewal to give rise to more stem cells, and the ability to differentiate into a variety of specialized daughter cells to perform specific functions[2].Stem cells (SCs) are found in the brain, peripheral blood,skeletal muscle, cutaneous tissue, bone marrow,liver and blood vessels[3].Recent evidence suggests that they are capable of self-renewal and have the responsibility to maintain and repair their tissue of origin [4]and have the potential to differentiate into different cell lineages with specialized functions.

\section{Materials and Method}

\subsection{Periodontal ligament stem cells culture}

Collect the healthy premolars extracted for orthodontic purposes and periodontally involved teeth with hopeless prognosis without periodontal treatment. Culture the hPDLCs and iPDLCs by trypsin digestion in vitro and observe their morphological characteristics with inverted microscope. The two kind of Periodontal ligament stem cells were cultured in petri dishs containing $\alpha$-MEM and $15 \%$ fetal bovine serum (FBS) and $1 \%$ antibiotics at $37^{\circ} \mathrm{C}$ and $5 \% \mathrm{CO} 2$. When cells adherent and grow up to 80-90\% confluent, wash twice with phosphate-buffered saline and detached from the culture surface using $0.25 \%$ trypsin-EDTA solution (hyclone, USA) to subculture cells steadily in line.

\subsection{Flow cytometric analysis}

Detect Surface Markers of stro-1, CD146 and CD45,CD34 in Mesenchymal Stem Cells by Flow Cytometry.

\subsection{The methyl thiazol tetrazolium (MTT) assay}

The third passage Cells were cultured and the proliferation ability of two cells with sdf- 1 in each group were evaluated using the methyl thiazol tetrazolium assay. It mainly convert yellow tetrazolium [3-(4, 5-dimethylthiazol-2-yl)-2, 5-diphenyltetrazolium bromide] to purple insoluble formazan crystals by MTT succinate reductase of mitochondrial respiratory chain that is active only in living cells. Replace the supernatant with $60 \mu \mathrm{L}$ of MTT solution and will found that Formazan crystals were formed after $1-1.5$ hours of incubation at $37^{\circ} \mathrm{C}$ and $5 \% \mathrm{CO} 2$. After dissolving crystals in 
$300 \mu \mathrm{L}$ of dimethyl sulfoxide, use ELISA Reader to read the absorbance at 490nm wavelength.Then, the rate of cell proliferation in healthy and periodontitis-affected groups induced by SDF-1 was assessed.

\subsection{Alizarin red staining}

Alizarin red staining to detect calcium nodules of hPDLCs and iPDLCs cultured for 14 days.

Collect the hPDLCs and iPDLCs with good growth status of induction after 14 days and remove the original culture medium from the culture dish, rinse three times with PBS and discard each dish. Add $2 \mathrm{ml}$ of 4\% paraformaldehyde to each dish for about 15 minutes and discard it. Then add 95\% ethanol $2.5 \mathrm{ml}$ until it is naturally air-dried. Add $0.1 \%$ alizarin red staining solution $2 \mathrm{ml}, 10 \mathrm{~min}$ after the complex then observe them under light microscope.

\subsection{Alkaline phosphatase (ALP) method}

Their abilities of proliferation and osteogenesis induced by different density of SDF-1 were evaluated by alkaline phosphatase (ALP). The well-grown third-generation iPDLSCs and hPDLSCs were digested and seeded in 96-well plates at a density of $2 \times 103$ cells per milliliter. After incubation for $24 \mathrm{~h}$, the supernatant was discarded and divided into two groups.Add $\alpha$-MEM culture medium containing $50 \mathrm{ng} / \mathrm{ml}, 200 \mathrm{ng} / \mathrm{ml}$ SDF-1 and SDF-1-free into the cells of iPDLSCs and hPDLSCs.The culture plates of iPDLSCs and hPDLSCs were detected on the 1st, 2nd and 3rd day respectively.The ALP activity of each well was measured by the enzyme - linked immunosorbent assay at $520 \mathrm{~nm}$.

Real-time quantitative PCR to investigate the expression of osteogenic relative genes of Runx2, OPN and OCN in hPDLCs and iPDLCs with SDF-1.

\section{RESULTS}

The stem cells of the primary periodontal ligament stem cells were successfully cultured in vitro and positive express stro-1, CD146 and the expression of CD34 and CD45 were negative. The proliferation ablity of hPDLCs and iPDLCs were increased and the expression of osteogenesis-related genes was up-regulated after SDF-1 induced. Compared with the control group, SDF-1 had the most significant effect on osteoblast differentiation of iPDLSCs at 50ng / ml, and 200ng / ml SDF-1 indicated the most effective affection of hPDLSCs.

\section{Conclusions}

1.We successfully isolated stem cells from the periodontium of both healthy and periodontitis-affected teeth.

2. SDF-1 has a certain effect on the osteogenic differentiation of periodontal ligament stem cells.

3. SDF-1 had different effects of periodontal ligament stem cells from different tissues.

4. Viability and proliferation ability of cells isolated from the periodontium of healthy teeth were significantly greater than those of cells isolated from the periodontitis-affected teeth.

Keywords: Periodontitis;Regeneration;Stem Cells;Stromal cell derived factor-1;health Periodontal ligament stem cells;inflammatory Periodontal ligament stem cells;osteogenic differentiation

\section{DISCUSSION}

Stem cell-based therapies is recognized as one of the most effective ways in the field of regenerative medicine. Human-beings try to find effective measures to realize regeneration of the damaged tissues from non-surgical mechanical debridement to periodontal surgery and pharmaceutical therapy[5].Regenerative stem cell therapy has created a lot of hope among scientists and physicians for finding more effective treatment strategies to reconstruct damaged periodontium[6]. Recent studies have reported that the chemokine SDF-1 and its receptor CXCR4 play an important role in stem cell migration, chemotaxis,homing and differentiation[7]. SDF-1 also 
significantly increased the chemotaxis of mesenchymal stem cells with increasing of the expression of CXCR4 in the mesenchymal stem cells transfected with CXCR4 gene[8], whereas inhibited mesenchymal stem cell pairs by blocking the expression of CXCR4[9].Therefore, apply SDF-1 to teeth tissue engineering to induce stem / progenitor cells to damaged periodontal groups weaving repair will be a promising development direction[10].

\section{References}

[1]. Kuula H, Salo T, Pirila E, et al. Local and systemic responses in matrix metalloproteinase 8-deficient mice during Porphyromonas gingivalis-induced periodontitis. Infect Immun, 2009, 77(2):850-859.

[2]. Menicanin Danijela, Mrozik Krzyszt of Marek.Periodontal-Ligament-Derived Stem Cells Exhibit the Capacity for Long-Term Survival, Self-Renewal, and Regeneration of Multiple Tissue Types in Vivo.Stem Cells Development. 2014, 23(9): 1001-1011.

[3]. Heino T. J., Hentunen T. A. Differentiation of osteoblasts and osteocytes from mesenchymal stem cells.Current Stem Cell Research and Therapy. 2008;3(2):131-145.

[4]. Gault P, Black A, R omette JL, et al. Tissue-engineered ligament:implant constructs for tooth replacement. J Clin Periodontol, 2010, 37(8):750-758.

[5]. Estrela C, Alencar AH, Kitten GT, Vencio EF,Gava E. Mesenchymal stem cells in the dental tissues:perspectives for tissue regeneration. Braz Dent J.2011;22(2):91-8.

[6]. Kalra N, Guruprasad CN, Naik S, Pradeep AR.Stem cells: a novel approach to periodontal regeneration. AOSR. 2011;1:116-21.

[7]. C. Hu, X. Yong, C. Li et al., "CXCL12/CXCR4 axis promotes mesenchymal stem cell mobilization to burn wounds and contributes to wound repair,” Journal of Surgical Research, vol. 183, no. 1, pp. 427-434, 2013.

[8]. Bhakta S,Hong P,The surface adhesion molecule CXCR4 stimulates mesenchymal stem cell migration to stromal cell derived factor-1 in vitro but does not decrease apoptosis under serum deprivation. Cardiovasc Revasc Med,2006,7(1):19-24.

[9]. Li s, Deng L. et al. Up-regulation of CXCR4 favoring neural-like cells migration via AKT activation[J].Neurosci Res,2010,67:293-299.

[10]. PejcicA,Kojovic D,et al.Stem cells for periodontal regeneration..BJMG.2013,16(1):7-12. 